\title{
A Tannic Acid-Inducible and Hypoviral-Regulated Laccase3 Contributes to the Virulence of the Chestnut Blight Fungus Cryphonectria parasitica
}

\author{
Hea-Jong Chung, ${ }^{1}$ Bo-Ra Kwon, ${ }^{2}$ Jung-Mi Kim, ${ }^{2}$ Seung-Moon Park, ${ }^{2}$ Jong-Kun Park, ${ }^{1}$ Byeong-Jin Cha, ${ }^{3}$ \\ Moon-Sik Yang, ${ }^{2}$ and Dae-Hyuk Kim ${ }^{2}$ \\ ${ }^{1}$ Division of Natural Sciences and Technology, Wonkwang University, Iksan, Chonbuk 570-749, Korea; ${ }^{2}$ Institute for Molecular \\ Biology and Genetics, Research Center of Bioactive Materials, Chonbuk National University, Jeonju, Chonbuk 561-756, \\ Korea; ${ }^{3}$ Department of Agricultural Biology, Chungbuk National University, Cheongju, Chungbuk 361-763, Korea
}

Submitted 23 June 2008. Accepted 12 August 2008.

\begin{abstract}
A new laccase gene (lac3) from the chestnut blight fungus Cryphonectria parasitica was induced by the presence of tannic acid, which is abundant in the bark of chestnut trees and is assumed to be one of the major barriers against pathogen infection. However, other commonly known laccase inducers, including ferulic acid, 2,5-xylidine, catechol, and $\mathrm{pH}$, did not induce lac3 transcription. Moreover, the hypovirus modulated the induction of lac 3 transcription, abolishing the transcriptional induction of the lac 3 gene by tannic acid. A functional analysis of lac3 using a lac3-null mutant indicated that fungal growth and other morphological characteristics, including pigmentation and sporulation, were not affected. However, a virulence assay indicated that the loss of function of a tannic acid-inducible and hypoviral-regulated laccase resulted in reduced virulence without detectable changes in the morphological features. The constitutive expression of lac3 resulted in no significant differences in the necrotic lesions from those caused by the wild type, but its expression in the presence of the hypovirus led to larger lesions than those caused by the hypovirulent strain. These results suggest that the lac3 gene product may not be the only determinant of fungal virulence in chestnut trees but is an important factor.
\end{abstract}

Additional keyword: hypovirulence.

The chestnut blight fungus Cryphonectria parasitica virtually eliminated American chestnut (Castanea dentata) in the early 20th century (Anagnostakis 1982, 1988). However, strains containing the double-stranded (ds)RNA virus Cryphonectria hypovirus1 show characteristic symptoms of reduced virulence, i.e., hypovirulence (Anagnostakis 1982; Milgroom and Cortesi 2004; Nuss 2005; Van Alfen et al. 1975), and display diverse hypovirulence-associated traits such as reduced pigmentation, sporulation, laccase production, and oxalate accumulation (Elliston 1985; Havir and Anagnostakis 1983; Rigling et al. 1989). Because the phenotypic changes in the

Corresponding author: Dae-Hyuk Kim; E-mail: dhkim@chonbuk.ac.kr; Telephone: +82-63-270-3440; Fax: +82-63-270-3345.

* The $e$-Xtra logo stands for "electronic extra" and indicates that Figures 4 and 5 are published in color online. fungal host following hypovirus infection are specific, albeit pleiotropic, it has been suggested that the hypovirus specifically disturbs one or several regulatory pathways (Nuss 1996; Shang et al. 2008). Accordingly, several genes have been identified as targets because they are specifically regulated by the presence of hypovirus (Chen et al. 1996; Deng et al. 2007b]; Gao and Nuss 1996; Kasahara and Nuss 1997; Park et al. 2004). Recent studies using DNA microarrays have revealed changes in the transcriptional profile of a fungal host as a result of hypovirus infection and have facilitated grouping the genes showing aberrant expression into several metabolic pathways (Allen and Nuss 2004; Allen et al. 2003; Deng et al. 2007a). Among the specific target pathways of the fungal life cycle, the reduced virulence to chestnut appears to be one of the major effects of the fungus-hypovirus interaction.

Measuring the virulence of forest fungal pathogens such as chestnut blight fungus to their native hosts is more difficult than measuring the virulence of grass or annual pathogens, not least because of the unavailability of corresponding host trees in areas such as North America. As a result, inoculation of dormant chestnut stems (Jaynes and Elliston 1980), excised bark (Lee et al. 1992), and other hosts such as Golden Delicious apple (Fulbright 1984) have been considered as alternatives for measuring fungal virulence. In addition, several approaches have been suggested as indirect methods to compare fungal virulence. The two best alternatives are the measurement of extracellular laccase activity in liquid culture and the Bavendamm assay, which measures the degree of oxidizing browning on tannic acid-supplemented plates, because they have a high correlation with virulence (Rigling et al. 1989).

Laccases are multinuclear copper-containing phenol oxidases (p-diphenol:oxygen oxidoreductase, EC 1.10.3.2) that catalyze the oxidation of $p$-diphenols with the concurrent reduction of dioxygen to water (Thurston 1994), although the actual substrate specificities of laccases are often broad and vary with the enzyme source. Laccases belong to a small group of enzymes known as large blue copper proteins or blue copper oxidases, characterized by four cupric $\left(\mathrm{Cu}^{2+}\right)$ ions that are coordinated such that each of the ion species (types 1, 2, and 3) is associated with a single peptide chain. Laccase is produced by various sources, including bacteria, fungi, insects, and higher plants (Dean and Eriksson 1994). Many fungi produce multiple laccases that are encoded by gene families (Kim et al. 1995; Kumar et al. 2003; Perry et al. 1993; Yaver and Golightly 
1996). Fungal laccases have been implicated in the pigmentation of fungal spores (Clutterbuck 1972; Smith et al. 1998), the development of fungal fruiting bodies (Leatham and Stahmann 1981), fungal pathogenicity (Geiger et al. 1986; Marbach et al. 1985; Rigling et al. 1989), lignin degradation (Hataka 1998; Ishihara 1980), vegetative growth (Sengupta and Mukherjee 1997), and phytoalexin conversion (Schouten et al. 2002). However, with the exception of the conidial laccase of Aspergillus nidulans (Clutterbuck 1972; Law and Timberlake 1980; Yelton et al. 1985) and the secretory laccase of Botrytis cinerea (Schouten et al. 2002), very few of these functions have been demonstrated experimentally.

Cryphonectria parasitica produces at least two laccase isoforms, constitutive extracellular laccase (lac1) and intracellular laccase $(l a c 2)$, both of which are modulated by the presence of hypovirus. Previous studies have demonstrated that the lac1null mutant shows no changes in virulence and have suggested the presence of a tannic acid-inducible laccase (lac3) (Kim et al. 1995). Tannic acid is a commercial form of a mixture of hydrolyzable galloyl tannins (Yumiko et al. 2003). Tannins, which are abundant in the bark of the chestnut tree, are water-soluble phenolic compounds with molecular weights between 500 and 3,000 Da that have special properties, such as the ability to precipitate alkaloids, gelatin, and other proteins (Griffin 1986). In this study, we characterized the tannic acid-inducible laccase and examined the biological functions of the lac3 gene.

\section{RESULTS}

Characteristics of the lac3 gene.

Computer-assisted alignment of multiple fungal laccases revealed several conserved regions with more than five consecutive identical amino acid residues. Using degenerate primers designed from conserved regions of fungal laccases (Fig. 1), an 833-bp DNA fragment was amplified, cloned, and sequenced. Sequence analysis of 40 clones led to the identification of two different clone types, one identical to the previous laccase 1 sequence (Choi et al. 1992; Rigling and Van Alfen 1991) and another that differs from the previous laccase 1 but is similar to other known fungal laccases (Fig. 1). A homology search of this new sequence revealed the highest similarity to a hypothetical gene of Magnaporthe grisea, sharing 36 and 59\% identity at the nucleotide and amino acid levels, respectively, but only 35 and $51 \%$ identity to lac 1 . Screening of a genomic $\lambda$ library, using the cloned polymerase chain reaction (PCR) product as a probe, resulted in three positive clones out of 40,000. A 4.7-kb SacI-digested fragment from the genomic $\lambda$ clone was subcloned (pGlac3F), sequenced, and found to contain the full-length $C$. parasitica laccase 3 gene, lac3. Based on the genomic sequence, reverse transcription (RT)-PCR was used to isolate a nearly full-length cDNA fragment, using primers that hybridize at nucleotide positions (nt) -3 to 24 and 2,151 to 2,179 , relative to the start codon. Sequence analysis of the cDNA obtained using RT-PCR indicated that the lac3 gene consists of seven exons, with six intervening sequences ranging in size from 65 to $100 \mathrm{bp}$ (lac3 GenBank accession number, AY994151). The deduced lac3 protein product (LAC3) consists of 567 amino acids, including an 18-residue secretory signal peptide typical of extracellular enzymes, which was predicted by the algorithm of Nielsen and associates (1997). The predicted unprocessed and mature protein products have estimated molecular masses of 62 and $60 \mathrm{kDa}$, with isoelectric points (pI) of 4.14 and 4.12, respectively. The deduced amino-acid sequence contains 11 putative N-glycosylation sites (Asn-Xxx-Ser/Thr) (Gavel and Von Heijne 1990), at nt positions 58, 69, 85, 112, 124, 234, 270, 342, 403, 422, and 545 (Fig. 1).
A primer-extension experiment revealed that the transcription start site of lac 3 is located $214 \mathrm{bp}$ upstream of the putative start codon. Sequence analysis of the first 1,939 bp of the promoter region of the lac 3 gene revealed putative CAAT and TATA boxes at nucleotides -204 and -89 , respectively, relative to the transcription start site. In addition, a putative consensus metal-response element, TGCRCNC, and a xenobiotic-response element, TNGCGTG, are present at 602 and 905 bp upstream of the first ATG, respectively. The region also contains the sequence TATCT, a putative binding site for an NIT2-like protein, which could be involved in nitrogen metabolite regulation (Jarai et al. 1992), as well as a consensus binding site, TCT46GCTG, for the copper-regulated transcription factor ACE1 (Gralla et al. 1991), at 893 and 470 bp upstream of the first ATG, respectively. The sequence around the first ATG is in good agreement with Kozak's consensus sequence in that the 3 position is the A in CATTATG. A putative consensus polyadenylation signal, AATATA (Kim et al. 2001), is present $240 \mathrm{bp}$ downstream of the stop codon.

All of the residues involved in $\mathrm{Cu}$ (II) ligand binding (ten histidine residues and one cysteine residue) that are conserved in fungal laccases (Kumar et al. 2003) are present in the lac3 coding sequence of $C$. parasitica (Messerschmidt 1997) (Fig. 1). Another residue (leu), 10 amino acids downstream of the conserved cysteine involved in the type-I $\mathrm{Cu}$ (II) binding site, is also preserved. However, the absence of the leu-glu-ala tripeptide motif adjacent to the last conserved histidine and the presence of the leucine residue rather than a phenylalanine residue suggests that LAC3 does not possess high ionization potential (Temp and Eggert 1999).

A comparison of the deduced amino-acid sequence of the lac3 gene product with those of other fungal laccases revealed that LAC3 shows higher similarity to laccases from other species than to LAC1 from the same species. The lac3 gene is most closely related to lac2 from Gaeumannomyces graminis var. graminis (47\% amino acid identity), followed by lac 2

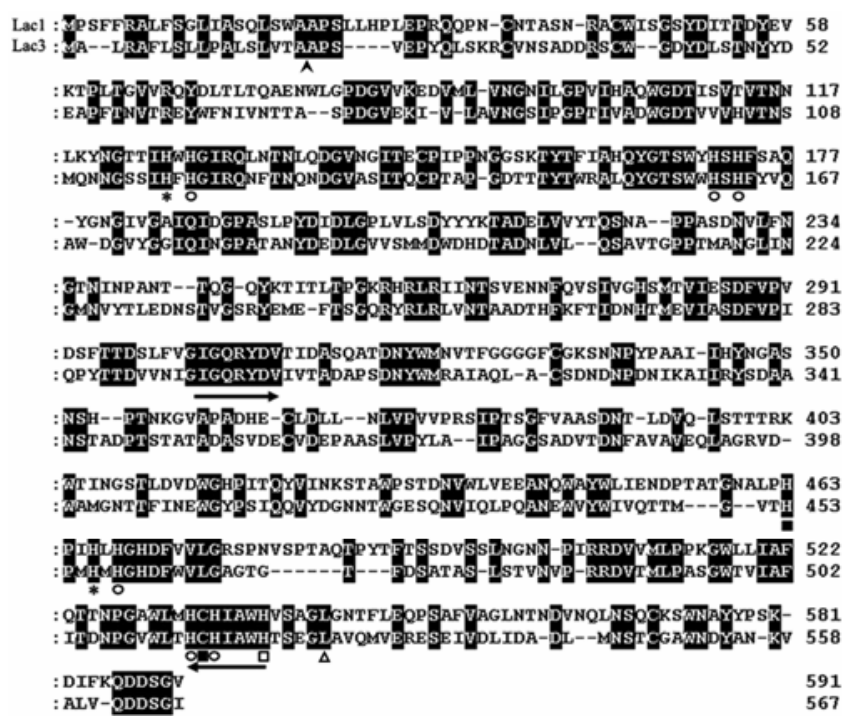

Fig. 1. Alignment of the deduced amino-acid sequences of Cryphonectria parasitica lac 1 (top) and lac3 (bottom). Identical residues are shaded, and dashes represent gaps in the aligned sequences. The putative cleavage site of the signal peptide is indicated by an arrowhead. The amino acid residues proposed to be involved in copper binding are indicated as follows: type 1 (squares), type 2 (asterisks), and type 3 (circles) (Messerschmidt 1997). The last conserved histidine and the leucine residue, 10 amino acids downstream of the conserved cysteine involved in the type $1 \mathrm{Cu}$ (II) binding site, are indicated by an open square and a triangle, respectively. Conserved regions used for primer design are indicated by arrows. 
from G. graminis var. tritici (46\%). The lac3 shows $41 \%$ identity to lac1 from $C$. parasitica.

\section{Regulation of lac3 gene expression.}

Previous studies have shown that the extracellular laccase activity in filtrates from cultures grown in standard growth conditions was due to laccase1, whereas a new laccase activity (laccase3) was detected in culture filtrates grown in the presence of tannic acid and showed different electromobility on native agarose gels (Kim et al. 1995). Similarly, Northern blot analysis showed no accumulation of the lac3 gene transcript under normal growth conditions, including EP complete liquid medium and solid media such as malt extract agar or potato dextrose agar containing $100 \mathrm{mg}$ of L-methionine and $1 \mathrm{mg}$ of biotin per liter (PDAmb). However, the accumulation of the lac3 gene transcript was induced in modified Bavendamm's medium, a tannic acid-supplemented malt extract agar (Rigling et al. 1989). Strong induction of the lac3 transcript on modified Bavendamm's medium was first observed $12 \mathrm{~h}$ after induction; the expression peaked at $24 \mathrm{~h}$ and then decreased gradually (Fig. 2). The concentration of tannic acid required for the induction was examined by testing $0.7,0.07$, and $0.007 \%$ (wt/vol) tannic acid; concentrations as low as $0.007 \%$ induced lac3 expression on modified Bavendamm's medium. Induction of the lac3 transcript was also observed on minimal medium containing $0.7 \%$ tannic acid. However, when the tannic acid in modified Bavendamm's medium was replaced with citric acid, no accumulation of the lac 3 transcript was detected. There-

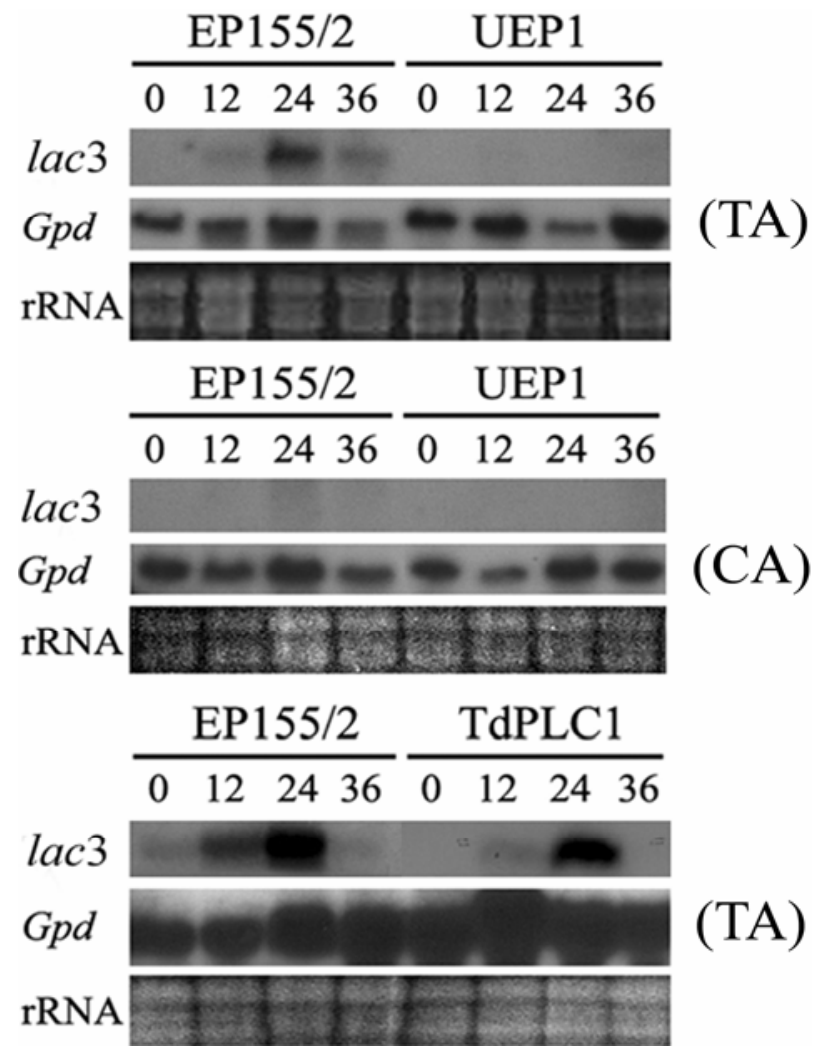

Fig. 2. Northern blot analysis of lac3 using the nearly full-length cDNA fragment as a probe. Total RNA was extracted from the strains grown on tannic acid (TA) - and citric acid (CA)-containing plates. Numbers indicate the hours after induction. Equal loading of RNA samples is demonstrated in the bottom panel, with a parallel blot that was hybridized with the glyceraldehyde-3-phosphate dehydrogenase gene $(G p d)$ probe as an internal control, and an ethidium bromide-stained gel (rRNA). The strains used, indicated above the panel, were the virus-free wild type (EP155/2), its isogenic virus-containing hypovirulent strain (UEP1), and the phohpolipase $\mathrm{C}$ gene cplc1-null mutant (TdPLC1). fore, its induction appears to be specific to the presence of tannic acid rather than other media components or growth conditions such as acidity. The lac 1 expression was also examined in different media containing tannic acid, but its induction was not observed (data not shown).

Because ferulic acid and 2,5-xylidine, aromatic compounds structurally related to lignin, are effective inducers of the production of other fungal laccases, their ability to induce the lac3 gene was examined. Using Northern blot analysis, the accumulation of the lac3 gene transcript was measured in both liquid and solid media containing either ferulic acid or 2,5-xylidine. No hybridizing lac3 band was detected, indicating that the expression of the lac3 gene was not induced by these ligninrelated aromatic compounds (data not shown). Other conditions known to induce other fungal laccases, such as $\mathrm{Mg}^{2+}$ and $\mathrm{Cu}^{2+}$ supplementation, were also tested, and no inducing effect on the lac 3 expression was observed.

Because the expression of a different $C$. parasitica laccase, lac 1 , is under the tight control of the phospholipase $\mathrm{C}$ gene cplc1 (Chung et al. 2006), we tested whether cplc1 modulates the induction of the lac3 gene. When a cplc1-null mutant was placed in induction conditions (medium containing tannic acid), the lac3 gene transcript was induced as in the wild type, indicating that the presence of the $c p l c 1$ gene is not necessary for the induction of the lac3 gene (Fig. 2).

We also examined whether an osmotic stress-involved mitogen-activated protein (MAP) kinase (cpmk1) (Park et al. 2004) and a mycelial growth- and conidiation-related MAP kinase (cpmk2) (Choi et al. 2005) affect the induction of the lac3 gene by placing both cpmk1- and cpmk2-null mutants in induction conditions. No changes in the induction of the lac3 transcript were observed in either MAP kinase mutant (data not shown), suggesting that the signaling pathways involving these MAP kinases are not responsible for the induction.

\section{Viral regulation of $l a c 3$ induction.}

Among the putative inducers that were tested, tannic acid was able to induce the lac 3 gene. To examine the viral regulation of lac3 induction, the lac 3 induction pattern in the isogenic hypovirulent strain UEP1 was compared with that in the wild-type strain EP155/2 (Fig. 2). Northern blot analysis of RNA from UEP1 cultured under induction conditions with tannic acid revealed no hybridizing lac 3 band, indicating that the hypoviral infection specifically prevented the induction of the lac3 transcript by tannic acid.

\section{Construction of a lac3-null mutant.}

To explore the effects of deletion of the lac3 gene, a lac3null mutant was constructed by site-directed recombination during integrative transformation. A linearized plasmid DNA containing the disrupted lac3 gene was used to transform the virus-free $C$. parasitica EP155/2. A total of 750 single-spore transformants were screened by PCR using outer and inner primers corresponding to $\mathrm{nt}-28$ to -11 and 2,190 to 2,210 , respectively, relative to the start codon (Fig. 3A). All but one transformant showed both 2.2- and 3.2-kb amplicons, corresponding to the wild-type and disrupted alleles of the lac 3 gene, respectively, suggesting that transformants were ectopic, resulting from random integrations of the transforming vector. However, one transformant did not contain the wild-type 2.2$\mathrm{kb}$ amplicon but showed only the $3.2-\mathrm{kb}$ fragment of the disrupted allele of the lac 3 gene, suggesting that the simple replacement of the wild-type allele with the disrupted allele occurred (data not shown). The putative lac3-null mutant (TdLAC3-63-6) was examined by Southern blot analysis (Fig. 3B). The hybridization pattern of BamHI- and XhoI-digested genomic TdLAC3-63-6 DNA with a probe corresponding to a 
1.1-kb XhoI fragment differed from that of the wild type, suggesting that the transforming vector integrated at the lac3 locus by site-directed homologous recombination (Fig. 3). Moreover, XhoI-digested TdLAC3-63-6 genomic DNA showed a hybridizing band of $4.6 \mathrm{~kb}$, corresponding to the expected size of the gene-replacement vector pDlac3 (Fig. 3A), and the 4.6$\mathrm{kb}$ band also hybridized to the 0.8-kb EcoRI/BamHI- $h$ ph fragment, indicating that the lac3 gene was replaced with part of the transforming vector rather than having been disrupted, as expected.

\section{Phenotypic characteristics of the lac3-null mutant.}

The mycelial growth rate of the lac3-null mutant was not changed on PDAmb in standard growth conditions (constant low-level light at $25^{\circ} \mathrm{C}$; Fig. 4). No difference in the growth rate as compared with that of the wild type was observed on minimal medium. However, when the lac3-null mutant was grown on minimal medium supplemented with tannic acid, the mycelial growth rate was significantly reduced to less than $55 \%$ of the radial growth of the wild type (Fig. 4). Surprisingly, even though the lac3 gene was strongly induced in the presence of tannic acid, no change in the growth rate was observed on tannic acid-containing modified Bavendamm's medium. In

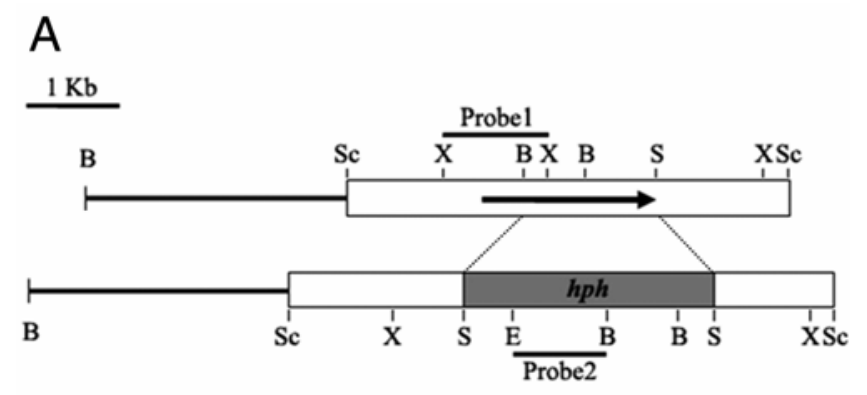

B

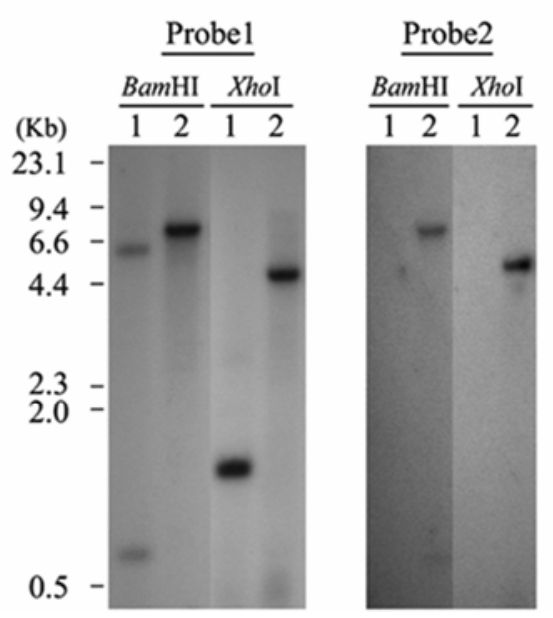

Fig. 3. Restriction and Southern blot analyses of the lac3-null mutant (TdLAC3-63-6) and the wild-type strain EP155/2. A, Restriction map of the lac3 genomic region and the gene-replacement vector pDlac3, which contains 1,890- and 1,370-bp fragments from the $5^{\prime}$ and $3^{\prime}$ flanking regions, respectively. Arrows indicate the direction of transcription. Boxes and lines indicate regions in which the sequences have or have not been determined, respectively. B, BamHI; E, EcoRI; S, SalI; ScI, SacI; and X, XhoI. B, Southern blot analysis of the wild-type EP155/2 strain (lane 1) and the cplc1-replaced transformant TdLAC3 (lane 2). All DNA samples were digested with BamHI or XhoI. The blots were probed with the 1.1-kb XhoI fragment (probe 1) and the 0.8-kb EcoRI/BamHI-hph fragment (probe 2). The TdLAC3-63-6 transformant has undergone the desired replacement at lac3, as evidenced by expected size changes in fragments that hybridized with probe 1 and the hybridization of the corresponding band with probe 2. The probes are indicated in the restriction map of the lac 3 gene in A. addition, no significant change in the size or darkness of the brown coloration was observed in the lac3-null mutant. Therefore, these results suggest that, although the protein product of the lac 3 gene is likely to be most important for $C$. parasitica to metabolize tannic acid, some other laccases or enzymes, such as laccase 1 or peroxidases, respectively, may also be responsible for the brown coloration on modified Bavendamm's medium.

To ensure that the phenotypic changes attributed to the lac3null mutant were due to lac3 gene replacement, the lac3-null mutant TdLAC3-63-6 was complemented in trans with a wildtype allele of the lac3 gene. When TdLAC3-63-6 was transformed with the lac 3 genomic clone, the defective growth on minimal medium containing tannic acid was corrected, confirming that the phenotypic changes in the lac3-null mutant were due to the specific deletion of the lac 3 gene.

The asexual sporulation of TdLAC3-63-6 was compared with that of the virus-free EP155/2 and virus-containing UEP1 strains. There was no significant difference in the asexual sporulation of TdLAC3-63-6 and EP155/2. The number of conidia produced by the hypovirulent UEP1 was significantly lower than that of the other two strains, as expected. No changes in pigmentation were caused by the loss of function of the lac3 gene (Fig. 4).

\section{Osmotic and temperature sensitivity of the lac3-null mutant.}

During cultivation under hypertonic conditions (PDAmb supplemented with $2 \mathrm{M}$ sorbitol) (Park et al. 2004), the lac3-

\section{EP155/2 TdLAC3 TcLAC3 UEP1}

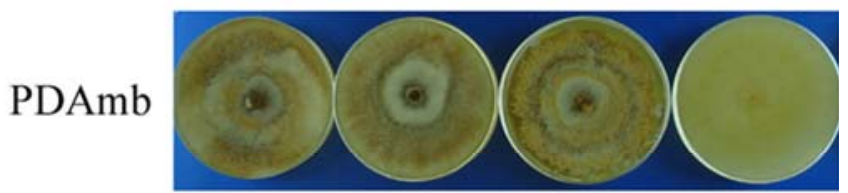

MM

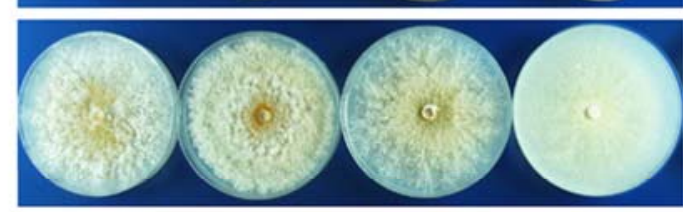

$\mathrm{MM}(+)$

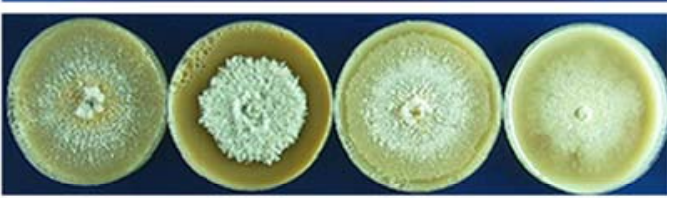

ME

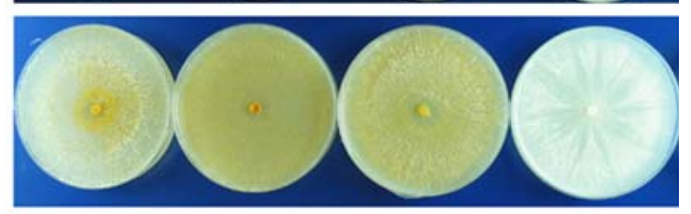

$\mathrm{ME}(+)$

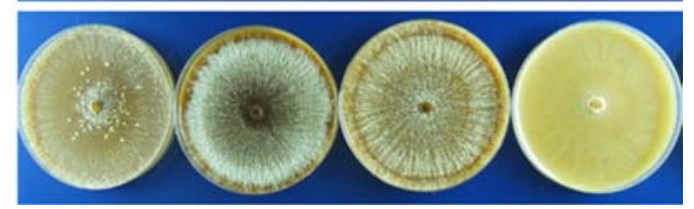

Fig. 4. Colony morphologies on different media. The colony morphologies of four strains after 14 days of cultivation on different media are shown. The growth media, indicated at the left, are potato dextrose agar containing $100 \mathrm{mg}$ of L-methionine and $1 \mathrm{mg}$ of biotin per liter (PDAmb), minimal medium (MM), and malt extract medium (ME); (+) indicates the inclusion of $0.7 \%$ tannic acid. The strains used, indicated above the panel, were the virus-free wild type (EP155/2), its isogenic virus-containing hypovirulent strain (UEP1), the lac3-null mutant (TdLAC3-63-3), and the lac3-complemented strain (TcLAC3). 
null mutant and the wild-type strain EP155/2 showed similar hyphal growth patterns. These results agree well with those of Northern blot analysis of the osmosensitive cpmk1-null mutant, which showed no changes in the induction of the lac3 gene. These facts strongly suggest that the loss of function of the lac 3 gene caused no changes in the osmotic sensitivity.

The temperature sensitivity of the lac3-null mutant was examined at various temperatures. No significant changes in relative growth rate were observed, suggesting that the mutant is not temperature sensitive.

\section{Virulence assay of the lac3-null mutant.}

In a virulence assay on excised chestnut tree bark (Lee et al. 1992), the size of the necrotic area induced on excised bark by the lac3-null mutant was smaller than that of the wild-type strain EP155/2, indicating that the lac3-null mutant is less virulent than EP155/2 (Table 1). The lac3-complemented strain of the mutant caused a necrotic area of size similar to that of the wild type, suggesting that the lac 3 gene is involved in determining the virulence of this fungus. However, the lac3null mutant produced a larger necrotic area than the hypovirulent strain UEP1, indicating that although the lac3 gene appears to be a pathogenic determinant, clearly other factors contribute to fungal pathogenicity as well.

\section{Characteristics and altered virulence of the lac 3 transformants.}

As an approach to modulate the expression of the lac 3 gene, a fusion construct replacing the lac 3 promoter with a constitutive and hypoviral-independent promoter (Kim et al. in press) was used to transform the wild-type strain EP155/2. The resulting transformant, T188::lac3-1, was then single-spored and used for coculture with the hypovirulent strain UEP1 to obtain a hypovirus-containing transformant, T188::lac3-1/CHV1. As shown in Figure 5, the transformant T188::lac3-1 was indistinguishable from the parental wild-type EP155/2 in conidiation, pigmentation, and colony morphology on PDAmb medium under standard growth conditions. Upon transfer of $\mathrm{CHV} 1$ through anastomosis, T188::lac3-1/CHV1 exhibited the cultural characteristics of UEP1. However, Northern blot analysis revealed the accumulation of the lac 3 transcript in the transformant T188::lac3-1 grown in EP complete medium, whereas no transcript was observed in the wild-type EP155/2, suggesting that the transformant T188::lac3-1 does not require tannic-acid induction for the expression of the lac 3 gene. Moreover, the hypovirus-containing transformant T188::lac3-1/CHV1 showed considerable accumulation of the lac 3 transcript, confirming that the lac 3 expression derived from the cryparin core promoter became constitutive rather than inducible in this transformant,

Table 1. Role of the Cryphonectria parasitica lac3 gene in virulence ${ }^{\mathrm{w}}$

\begin{tabular}{|c|c|c|c|}
\hline Strain or mutant & lac3 genotype $\mathrm{e}^{\mathrm{x}}$ & CHV1 $^{y}$ & Canker $\operatorname{area}^{\mathrm{z}}$ \\
\hline EP155/2 & + & - & $123.2 \pm 5.7 \mathrm{a}$ \\
\hline UEP1 & + & + & $76.3 \pm 2.2 \mathrm{~b}$ \\
\hline TdLAC3-63-3 & - & - & $98.3 \pm 1.9 \mathrm{c}$ \\
\hline TcLAC3 & + & - & $120.1 \pm 11.3 \mathrm{a}$ \\
\hline T188::lac3-1 & $\mathrm{C}$ & - & $131.4 \pm 21.7 \mathrm{a}$ \\
\hline T188::lac3-1/CHV1 & $\mathrm{C}$ & + & $103.0 \pm 4.1 \mathrm{c}$ \\
\hline
\end{tabular}

${ }^{\text {w }}$ Virulence was measured using excised chestnut tree bark, as described previously (Lee et al. 1992). Three replicates for each strain were used, and each experiment was repeated three times. Lesion measurements are shown as mean \pm standard deviation $\left(\mathrm{mm}^{2}\right)$.

${ }^{x}+$ and - indicate the presence or absence of the lac 3 gene, respectively. C indicates the constitutive expression of the lac 3 gene.

${ }^{\mathrm{y}}+$ and - indicate the presence or absence of CHV1, respectively.

${ }^{\mathrm{z}}$ Values followed by the same letter are not significantly different $(P<$ 0.05 ), as determined by Duncan's multiple-range test. and its expression was not affected by the presence of CHV1. Examination of the laccase activities in culture filtrates showed that the enzyme activity of the transformant T188::lac3-1 was higher than that of EP155/2. In addition, the hypovirus-containing transformant T188::lac3-1/CHV1 showed higher laccase activities than UEP1, in good agreement with the Northern blot results. These results indicate that the modulation of lac 3 gene expression resulted in no detectable changes in the morphological characteristics of the fungus. In addition, the lac 3 induction in response to tannic-acid supplementation appeared to be determined by the promoter activity at the transcription

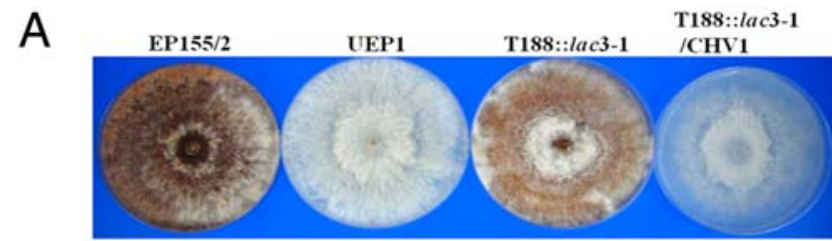

B
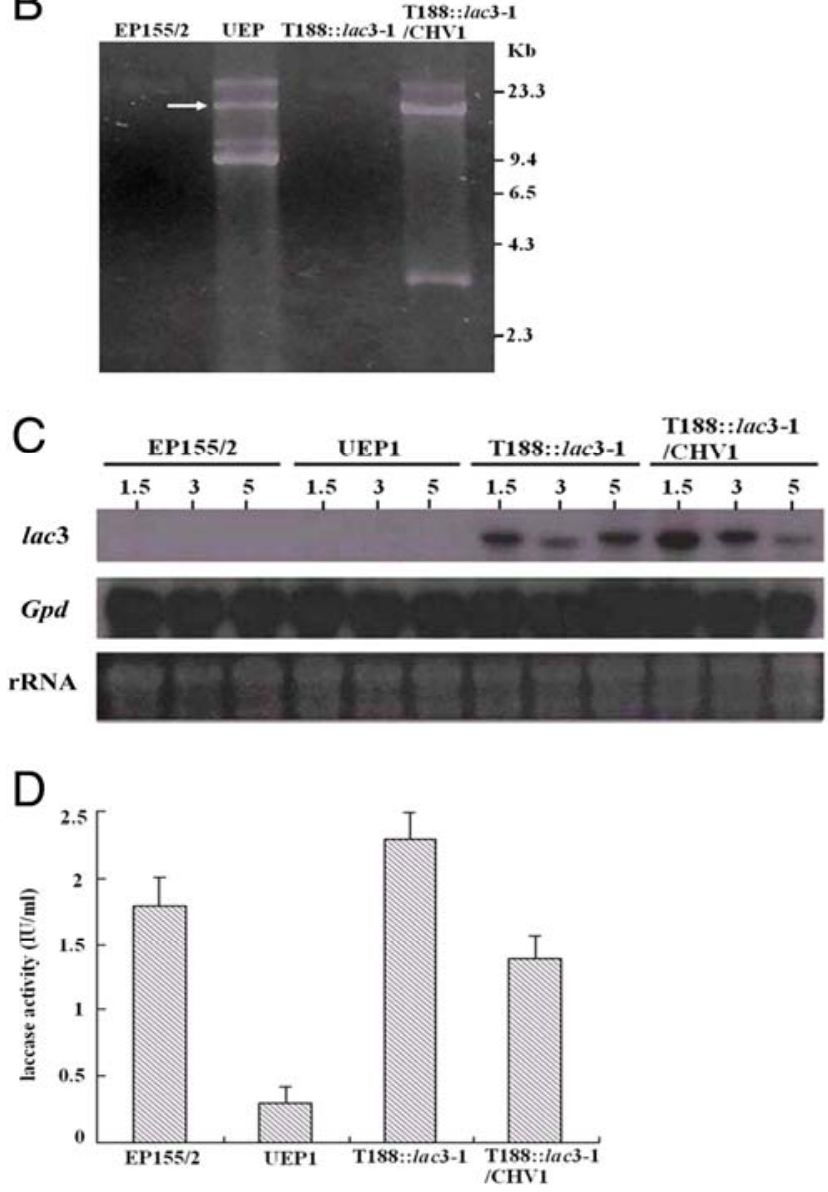

Fig. 5. Characteristics of transformants showing modulated expression of the lac3 gene. A, Colony morphology on potato dextrose agar containing $100 \mathrm{mg}$ of L-methionine and $1 \mathrm{mg}$ of biotin per liter (PDAmb). B, Ethidium bromide-stained gel showing the characteristic 12.7-kb genome of CHV1. The arrow indicates the viral genome of CHV1. C, Northern blot analysis of lac3 expression. Total RNA was extracted at the times indicated in hours after inoculation. Equal loading of RNA samples is shown with a parallel blot that was hybridized with the glyceraldehyde-3phosphate dehydrogenase gene $(G p d)$ probe as an internal control. An ethidium bromide-stained gel (rRNA) is also shown. D, Laccase activities of culture filtrates of transformants. The laccase activity was determined in filtered media harvested 3 days after inoculation in EP complete medium. The strains used, indicated above the panel, were the virus-free wild type (EP155/2), its isogenic hypovirus-containing hypovirulent strain (UEP1), the constitutive lac3-expressing transformant (T188::lac3-1), and the hypovirus-containing lac3 transformant (T188::lac3-1/CHV1). 
level, and the increased laccase activities in culture filtrates of the transformants suggested that the protein product of the lac3 gene is secreted as an extracellular enzyme.

In the virulence assay, no significant difference in lesion size was observed between the transformant T188::lac3-1 and its parental wild-type strain EP155/2. However, the hypoviruscontaining transformant T188::lac3-1/CHV1 did cause a larger lesion than the hypovirulent strain UEP1 but not as large as that caused by the wild-type (Table 1). These results suggest that the constitutive expression of the lac3 gene alone did not further increase the fungal virulence of the wild-type EP155/2. However, when the expression of the lac3 gene was severely hampered by the presence of CHV1, such as in the hypovirulent strain UEP1, the restoration of lac 3 expression made it possible to enhance the fungal virulence. Therefore, it appears that the expression of the lac 3 gene may not be necessary and sufficient as a single determinant of virulence but its expression at a certain level contributes to the maintenance of wildtype fungal virulence.

\section{DISCUSSION}

At least three laccases were predicted to be present in $C$. parasitica (Kim et al. 1995). Two are extracellular laccases, one constitutive (lac1) and the other tannic acid-inducible (lac3), and one is an intracellular laccase (lac2) (Kim et al. 1995). In this study, a new laccase-encoding gene and its corresponding cDNA were isolated from $C$. parasitica. Based on the sequence divergence from the $l a c 1$ gene structure having a canonical secretory signal peptide and tannic acid induction, the new laccase gene appeared to be the lac3 gene. In lac3, the single cysteine residue and 10 histidine residues that are required to bind the four catalytic cupric ions in all blue copper oxidases are conserved. Studies of type-I copper centers have shown that an additional residue 10 amino acids downstream of the conserved cysteine residue has a major effect on the redox potential of the cupric ion (Temp and Eggert 1999). This residue is a leucine residue in the lac 3 gene, but phenylalanine and methionine residues are present in the laccase sequences of other fungi and plants (Jarai et al. 1992). Based on its postulated redox potential (Eggert et al. 1998), the protein product of the lac3 gene corresponds to a class 2 laccase. The Aspergillus nidulans laccase, which contains a methionine residue at the site discussed above, belongs to class 1 and exhibits a very low similarity to all other known laccases, reflecting the very specialized function of spore morphogenesis in this fungus. In addition, the Pycnoporus cinnabarinus laccase, with a phenylalanine residue at the crucial site, belongs to class 3 and appears to be the only phenoloxidase but not a lignin peroxidase or manganese peroxidase; a single isoform is produced under conditions of lignin degradation. These results demonstrate the variety of specialized physiological functions of laccases. Interestingly, the deduced sequence of the previously characterized C. parasitica laccase 1 revealed only $56 \%$ similarity to laccase 3 at the amino-acid level but both are class 2 laccases, suggesting that the enzymes have a similar enzymatic function in different environments, whether or not tannic acid is present.

Tannic acid is a strong laccase 3 inducer. Fungi are diverse in their responses to putative laccase inducers, and differences in the regulation of gene transcription may reflect different physiological functions for fungal laccases. Most well-known inducers are toxic compounds that could resemble either breakdown products of lignin or antimicrobial agents secreted by hosts or competing fungi. Thus, the elicitation effect most likely represents a response to eliminate such toxic compounds by enzymatic transformation. However, some fungi may not require such an enhanced defense reaction, e.g., the ligninolytic basidiomycete PM1, in which laccase activity is not stimulated by several putative laccase inducers (Coll et al. 1993). Likewise, in $C$. parasitica, elicitation was not observed with other wellknown lignin-related inducers but instead by tannic acid, which is abundant in the bark of the chestnut tree (Griffin 1986). These results are in good agreement with the fact that $C$. parasitica is not a wood-decaying fungus but a canker-causing fungus, which is not involved in lignin degradation but must deal with the natural host barrier of tannic acid.

At least three laccases are present in C. parasitica and all appear to be under the control of complex but specific regulation (Kim et al. 1995). Previous studies have shown that both positive and negative pathways are involved in the modulation of lac 1 expression (Larson et al. 1992). Moreover, the $\mathrm{IP}_{3} / \mathrm{Ca}^{2+}$ second-messenger system regulates the viral-specific stimulatory pathway of lac 1 expression, and the negative pathway appears to depend on ongoing protein synthesis for the proteinaceous inhibitory factor (Larson et al. 1992). In addition, the presence of the wild-type $c p l c 1$ is a prerequisite for both proper expression and modulation of the lac 1 transcript (Chung et al. 2006). Our results indicate that the expression of the lac1 gene is unaffected by the presence of tannic acid, and $c p l c 1$, which is necessary to mediate the appropriate expression of the lac 1 gene, is not required for the induction of the lac3 gene by tannic acid. Considering the different responsiveness to tannic acid and differences in the requirement for the PLC gene, we propose the following model for the regulatory pathways that govern the expression of $C$. parasitica laccases (Fig. 6). There are at least two regulatory pathways that regulate the expression of laccases, and the expression of each laccase is governed independently by the corresponding specific pathway. Given that laccase 1 and laccase 3 each has its own specific regulatory pathway, it is of interest to see how the hypovirus can regulate both specific regulatory pathways. The small genome size of CHV1-713 and the efficient use of the viral genome suggest that, although the regulatory pathways for laccase expression are independent, there may be a common denominator in appropriate laccase expression, which could be the primary target of the viral regulation of fungal laccase expression. It is possible that this common denominator is the beginning component in a regulatory hierarchy, although the opposite regulatory cascade is still possible. This hypothesis of distinct regulation mechanisms for laccase expression suggests different physiological roles for these enzymes. However, complicating the general gene regulation-function relationship, the induction of a specific laccase gene does not necessarily have a beneficial effect on fungal fitness. In Botrytis cinerea, the laccase Bclcc 2 is strongly expressed in the presence of either tannin or resveratrol, a phytoalexin from grapevine;

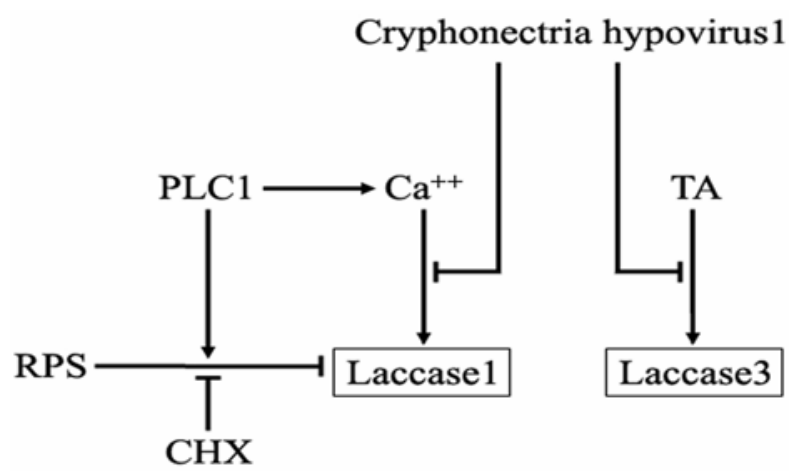

Fig. 6. A possible model of the regulatory pathway governing the modulation of hypoviral-specific laccases. Arrows indicate stimulatory or essential pathways, and lines with a bar at the end indicate inhibitory pathways for laccase expression. RPS = proteinaceous inhibitory factors, and $\mathrm{CHX}=$ cycloheximide. 
however, the protein product of Bclcc2 did not detoxify resveratrol, but rather, converted it into compounds that are more toxic to the fungus, resulting in self-intoxication (Schouten et al. 2002).

Although fungal laccases participate in a variety of physiological processes, they can be grouped into three major biological processes, i.e., lignin metabolism, fungal morphogenesis, and defense reactions. Defense reactions in a living host can be categorized as protection against host oxidative responses, detoxification of adverse phenolic compounds, and pigmentation (Hoegger et al. 2004). Based on the strong induction of the lac 3 transcript by tannic acid, which is abundant and assumed to be one of the major barriers against pathogen infection, it was unexpected that the lac3-null mutant showed no significant changes in colony morphology or brown coloration when cultured on a tannic acid-containing malt extract medium, Bavendamm's medium. However, deletion analysis of lac3 indicated that LAC3 is involved in mycelial growth under stressed conditions, such as on minimal medium containing supplemented tannic acid. Because the lac3 gene is induced by tannic acid and is required for active growth in nutrient-poor conditions, which may be reminiscent of chestnut bark, the biological function of the lac 3 gene seems to be defense against the host barrier and facilitates the fitness of the pathogenic $C$. parasitica in the hostile tannin-rich environment of chestnut bark. This speculation agrees well with the fact that the lac3-null mutant caused a smaller necrotic area on excised chestnut bark than did the wild type. In addition, the virulence assay indicated that, although the constitutive expression of lac 3 by itself did not increase fungal virulence, its expression in the presence of the hypovirus was able to restore fungal virulence to an intermediate level. Therefore, lac 3 may not be necessary and sufficient for virulence, but it is an essential factor for $C$. parasitica to maintain an appropriate level of virulence in response to the host defensive barrier.

\section{MATERIALS AND METHODS}

\section{Fungal strains and growth conditions.}

The CHV1-713-containing hypovirulent $C$. parasitica UEP1 and its isogenic virus-free strain EP155/2 (ATCC 38755) were maintained on PDAmb at $25^{\circ} \mathrm{C}$ under constant low-level $(<2,000 \mathrm{l} \times)$ light (Kim et al. 1995). The preparation of the primary inoculum for liquid and plate cultures and the culture conditions were as described (Kim et al. 1995).

Radial growth on plates was assessed by measuring colony diameters. To determine biomass, mycelia were filtered through Miracloth (Calbiochem, La Jolla, CA, U.S.A.), were washed with sterile distilled water, were blotted between dry paper towels until no excess water emerged, were weighed to obtain the fresh weight, and were further dried at $80^{\circ} \mathrm{C}$ for 2 days, as described previously, to obtain the dry weight (Kim et al. 1995).

The acute induction of the lac 3 gene was performed as follows. Ten agar plugs $(0.5 \mathrm{~cm}$ diameter $)$ containing actively growing young hyphae were inoculated on cellophane that was layered on the top of PDAmb medium and were incubated until the mycelia reached the end of the petri plate. The cellophane and the actively growing $C$. parasitica were then transferred to a plate containing an appropriate concentration of inducing materials. To determine the effect of tannin on lac 3 induction, tannic acid (Sigma, St. Louis) was supplemented as described previously (Rigling and Van Alfen 1993). Likewise, the induction of the lac3 gene by aromatic compounds and copper was measured using 2,5-xylidine and ferulic acid (Bollag and Leonowicz 1984; Collins and Dobson 1997; Soden and Dobson 2001; Yaver and Golightly 1996) and $\mathrm{CuSO}_{4}$ (Giardina et al. 1999; Palmieri et al. 2000), respectively, as described. Citric acid was used to determine the effect of acidity on lac3 expres- sion (Kim et al. 2001). For chronic induction of the lac3 gene, growth media containing corresponding components were inoculated with an appropriate strain and were incubated under standard culture conditions.

\section{Isolation and characterization}

of an inducible laccase gene, lac 3 .

Degenerate primers specific for consensus nucleotide sequences corresponding to the most conserved amino acids in the inducible laccases were designed. The primers used were LAC3 (forward) 5'-GGNCARAGRTAYGAYGTN-3' and LAC3 (reverse) 5'-RTGCCAKGCRATRTGGCA-3'. PCR was conducted as described previously (Kim et al. 2002). The 833-bp PCR amplicon was inserted into the pGEM-T vector (Promega, Madison, WI, U.S.A.). The inserted DNA fragments of positive bacterial clones were sequenced using the dideoxynucleotide method and were analyzed before being used as hybridization probes for genomic $\lambda$ library screening according to a previously described procedure (Kim et al. 2002).

To obtain the lac3 cDNA clone, PCR using reverse transcriptase was performed with the cLAC3-F1 (forward) $5^{\prime}$ TCTCGTCATTATGGCCCT-3' and cLAC3-R1 (reverse) 5'TATGCCCGAATCGTCCTGCAC-3' primers. The cDNA was sequenced using the dideoxynucleotide method and synthetic oligonucleotide primers.

A primer extension experiment was performed to identify the transcriptional initiation site. The reverse primer 5'CTGCGGTCATCTGCCGAGTTGAGA-3' was used along with standard methods (Kim et al. 2002).

\section{Southern and Northern blot analysis.}

Genomic DNA from $C$. parasitica was extracted as described (Churchill et al. 1990). DNA $(10 \mu \mathrm{g})$ was digested with restriction enzymes, was blotted onto a nylon membrane, and was hybridized with radioactively labeled probes.

RNA from liquid cultures was extracted as described (Kim et al. 1995). RNA from solid cultures was prepared from mycelial mats grown on cellophane that was layered on top of appropriate medium as described previously (Park et al. 2004). Northern blot analysis was conducted as described (Kim et al. 1995). The levels of lac3 transcript in C. parasitica were assessed using the glyceraldehyde-3-phosphate dehydrogenase gene as the internal control (Choi and Nuss 1990).

\section{Construction of a replacement vector and fungal transformation.}

The replacement vector pDlac3, which was designed to favor double-crossover integration events, was constructed as follows. The 5.0-kb SacI fragment containing the $5^{\prime}$ and $3^{\prime}$ flanking regions of lac3 was ligated into SalI-inactivated pBluescriptII SK (+), and the resulting plasmid was used as a template for inverse PCR, using the primers 5'-GGTCGACCACCGTAGA CGCCGTCCCA-3' and 5'-GGTCGACCAAACGGAGAGAG AAGAG-3'. The primers were modified to incorporate the restriction site for SalI (underlined). The PCR amplicon was digested with SalI and religated before transformation into Escherichia coli. The resulting plasmid was digested further with SalI and was inserted along with the 2.4-kb SalI fragment of pDH25 (Cullen et al. 1987), which carries the hygromycin phosphotransferase gene $(h p h)$ cassette. In the replacement vector pDlac3, the $h p h$ cassette was inserted between nt 759 and 2,208 of the lac3 gene, relative to the start codon, and was flanked by approximately 1,890 and $1,370 \mathrm{bp}$ of the $5^{\prime}$ and $3^{\prime}$ end sequences, respectively. The SacI-digested insert fragment of pDlac3 was then used to transform the virus-free EP155/2 strain.

Functional complementation of the lac3-null mutant was carried out using the wild-type allele. The complementing vec- 
tor pRlac3 was constructed by insertion of the 2.6-kb HindIII fragment of $\mathrm{pBen}^{\mathrm{r}}$ (Chung et al. 2006) into the HindIII-digested pLac3, which contains the full-length lac3 gene, in pBluescriptII $\mathrm{KS}(+)$. The resulting vector was then used to transform the lac3-null mutant.

Protoplast preparation and transformation were performed as described previously (Churchill et al. 1990; Kim et al. 1995, 2002). PCR and Southern blot analyses were conducted on the genomic DNA of the transformants to screen for gene replacement and trans-complementation of the lac3 gene.

\section{Construction of a vector for constitutive and hypoviral-independent expression of the lac 3 gene.}

To modulate the expression pattern of the lac 3 gene, the core promoter of the cryparin gene was selected, because it is a strong constitutive promoter and is not affected by the presence of hypovirus (Kim et al. in press). The strong core promoter of the cryparin gene, consisting of the fragment between $n t-188$ and the translation start codon, was used to replace the corresponding endogenous lac3 promoter. The expression vector pCrylac3 was constructed using an overlap extension PCR method (Ge and Rudolph 1997). Briefly, the 188-bp PCR fragment of the core promoter was obtained from pCEB7 with the primers pCry-F (forward: 5'-GGGAGCTCTCGAGACAGGA C-3') and pCry-R (reverse: 5'-GAAGGGCCATTTTGATTGA AGTTTGGAGGG-3'). The primers plac3-F (forward: 5'-AAT CAAAATGGCCCTTCGAGCCTTT-3') and plac3-R (reverse: 5'-TTGAGCTCTTATATGCCCGAATCGTC-3') were used to amplify the lac3 gene. The underlined sequences indicate the junction between the core promoter and the lac 3 gene.

\section{Transmission of the dsRNA virus.}

Virus transmission was performed as previously described (Cortesi et al. 2001). Briefly, mycelial plugs of the virus-containing strain UEP1 were placed on PDAmb medium adjacent to mycelial plugs of the virus-free recipient transformants. After 10 days of coculture, putatively fused mycelia at the border between each pair of strains were transferred to fresh PDAmb and were incubated until they presented a sector that showed viral symptoms, such as reduced pigmentation. Mycelia in the low-pigmented region were transferred to benomyl-containing medium to select for the virus-infected recipient transformants. Strains were single-spored, and the presence of hypovirus was confirmed by the purification of dsRNA from the single-spored isolates.

\section{Characterization of the lac3-null mutant.}

The phenotypic and molecular characteristics of the cplc1null mutant were compared with those of the wild-type EP155/2 and hypovirulent UEP1 strains.

Phenotypic changes in pigmentation, conidiation, and mating capability were measured as described (Kim et al. 1995). Responses to chronic hyperosmotic stress and temperature sensitivity were also measured as described (Chung et al. 2006; Park et al. 2004). A virulence test using excised chestnut tree bark was conducted as described (Lee et al. 1992). Laccase activity was judged based on the coloring of the modified Bavendamm's medium (0.7\% tannic acid, $1.5 \%$ malt extract, and $2.0 \%$ agar) on which the strains were grown (Rigling et al. 1989), and activity in the culture fluid was determined by a spectrophotometric assay using 2,6-dimethoxyphenol as the substrate (Rigling et al. 1989).

\section{ACKNOWLEDGMENTS}

This work was supported by a Korea Science and Engineering Foundation (KOSEF) grant funded by the Korean Government (MOST) (R01-
2007-000-10369-0). We thank the Research Center of Bioactive Materials and the Research Center for Industrial Development of BioFood Materials at Chonbuk National University for kindly providing the facilities for this research.

\section{LITERATURE CITED}

Allen, T. D., and Nuss, D. L. 2004. Specific and common alterations in host gene transcript accumulation following infection of the chestnut blight fungus by mild and severe hypoviruses. J. Virol. 78:4145-4155.

Allen, T. D., Dawe, A. L., and Nuss, D. L. 2003. Use of cDNA microarrays to monitor transcriptional responses of the chestnut blight fungus Cryphonectria parasitica to infection by virulence-attenuating hypoviruses. Eukaryot. Cell 2:1253-1265.

Anagnostakis, S. L. 1982. Biological control of chestnut blight. Science 215:466-471

Anagnostakis, S. L. 1988. Cryphonectria parasitica, the cause of chestnut blight. Adv. Plant Pathol. 6:123-136.

Bollag, J. M., and Leonowicz, A. 1984. Comparative studies of extracellular fungal laccase. Appl. Environ. Microbiol. 48:849-854.

Chen, B., Gao, S., Choi, G. H., and Nuss, D. L. 1996. Extensive alteration of fungal gene transcript accumulation and elevation of G-protein-regulated cAMP levels by a virulence-attenuating hypovirus. Proc. Natl. Acad. Sci. U.S.A. 93:7996-8000.

Choi, E. S., Chung, H. J., Kim, M. J., Park, S. M., Cha, B. J., Yang, M. S., and Kim, D. H. 2005. Characterization of the ERK homologue CpMK2 from the chestnut blight fungus Cryphonectria parasitica. Microbiology 151:1349-1358.

Choi, G. H., and Nuss, D. L. 1990. Nucleotide sequence of the glyceraldehyde-3-phosphate dehydrogenase gene from Cryphonectria parasitica. Nucleic Acids Res. 18:5566.

Choi, G. H., Larson, T. G., and Nuss, D. L. 1992. Molecular analysis of the laccase gene from the chestnut blight fungus and selective suppression of its expression in an isogenic hypovirulent strain. Mol. PlantMicrobe Interact. 5:119-128.

Chung, H. J., Kim, M. J., Lim, J. Y., Park, S. M., Cha, B. J., Yang, M. S., and Kim, D. H. 2006. A gene encoding phosphatidyl inositol-specific phospholipase $\mathrm{C}$ from Cryphonectria parasitica modulates the lac1 expression. Fung. Genet. Biol. 43:326-336.

Churchill, A. C. L., Ciufetti, L. M., Hansen, D. R., Van Etten, H. D., and Van Alfen, N. K. 1990. Transformation of the fungal pathogen Cryphonectria parasitica with a variety of heterologous plasmids. Curr. Genet. 17:25-31.

Clutterbuck, A. J. 1972. Absence of laccase from yellow spored mutants of Aspergillus nidulans. J. Gen. Microbiol. 70:423-435.

Coll, P. M., Fernandez, J. M., Villanueva, J. R., Santamaria, R., and Perez, P. 1993. Purification and characterization of a phenoloxidase (laccase) from the lignin-degrading basidiomycete PM1(CECT 2971). Appl. Eviron. Microbiol. 59:2607-2613.

Collins, P., and Dobson, A. 1997. Regulation of laccase gene transcription in Trametes versicolor. Appl. Environ. Microbiol. 63:3444-3450.

Cortesi, P., McCulloch, C. E., Song, H., Lin, H., and Milgroom, M. G. 2001. Genetic control of horizontal virus transmission in the chestnut blight fungus, Cryphonectria parasitica. Genetics 159:107-118.

Cullen, D., Leong, S. A., Wilson, L. J., and Henner, D. J. 1987. Transformation of Aspergillus nidulans with the hygromycin-resistance gene, hph. Gene 57:21-26.

Dean, J. F. D., and Eriksson, K. E. 1994. Laccase and the deposition of lignin in vascular plants. Holzforschung 18:21-33.

Deng, F., Allen, T. D., Hillman, B. I., and Nuss, D. L. 2007a. Comparative analysis of alterations in host phenotype and transcript accumulation following hypovirus and mycoreovirus infections of the chestnut blight fungus Cryphonectria parasitica. Eukaryot. Cell 6:1286-1298.

Deng, F., Allen, T. D., and Nuss, D. L. 2007b. Ste12 transcription factor homologue CpST12 is down-regulated by hypovirus infection and required for virulence and femaale fertility of the chestnut blight fungus Cryphonectria parasitica. Eukaryot. Cell 6:235-244.

Eggert, C., Lafayette, P. R., Temp, U., Eriksson, K. M. N., and Dean, J. F. D. 1998. Molecular analysis of a laccase gene from the white rot fungus Pycnoporus cinnabarinus. Appl. Environ. Microbiol. 64:1766-1772.

Elliston, J. E. 1985. Characteristics of dsRNA-free and dsRNA-containing strains of Endothia parasitica in relation to hypovirulence. Phytopathology 75:151-158.

Fulbright, D. W. 1984. Effect of eliminating dsRNA in hypovirulents Endothia parasitica. Phytopathology 74:722-724.

Gao, S., and Nuss, D. L. 1996. Distinct roles for two G proteins alpha subunits in fungal virulence, mophology, and reduction revealed by targeted gene disruption. Proc. Natl. Acad. Sci. U.S.A. 93:14122-14127.

Gavel, Y., and Von Heijne, G. 1990. Sequence differences between glyco- 
sylated and non-glycosylated Asn-X-Thr/Ser acceptor sites: Implication for protein engineering. Protein Eng. 3:433-442.

Ge, L., and Rudolph, P. 1997. Simultaneous introduction of multiple mutations using overlap extension PCR. Biotechniques 22:28-30.

Geiger, J. P., Nicole, M., Nandris, D., and Rio, B. 1986. Root rot disease of Hevea brasiliensis I. Physiological and biochemical aspects of aggression. Eur. J. Pathol. 16:22-37.

Giardina, P., Palmieri, G., Scaloni, A., Fontanella, B., Faraco, V., Cennamo, G., and Sannia, G. 1999. Protein and gene structure of a blue laccase from Pleurotus ostreatus. J. Biochem. 341:655-663.

Gralla, E. B., Thiele, D. J., Silar, P., and Valentine, J. S. 1991. ACE1, a copper-dependent transcription factor, activates expression of the yeast copper, zinc superoxide dismutase gene. Proc. Natl. Acad. Sci. U.S.A. 88:8558-8562.

Griffin, G. J. 1986. Chestnut blight and its control. Hortic. Rev. 8:291-335.

Hataka, A. 1998. Lignin-modifying enzymes from selected white-rot fungi: Production and role in lignin degradation. FEMS (Fed. Eur. Microbiol. Soc.) Microbiol. Rev. 13:125-135.

Havir, E. A., and Anagnostakis, S. L. 1983. Oxalate production by virulent but not by hypovirulent strains of Endothia parasitica. Physiol. Plant Pathol. 23:369-376.

Hoegger, P. J., Navarro-Gonzalez, M., Kilaru, S., Hoffmann, M., Westbrook, E. D., and Kues, U. 2004. The laccase gene family in Coprinopsis cinerea (Coprinus cinereus). Curr. Genet. 45:9-18.

Ishihara, T. 1980. The role of laccase in lignin biodegradation. Pages 1731 in: Lignin Degradation: Microbiology, Chemistry and Potential Application. T. K. Kirk, T. Higuchi, and H. Chang, eds. CRC press, Boca Raton.

Jarai, G., Truong, H. N., Daniel-Vedele, F., and Marzluf, G. A. 1992. NIT2, the nitrogen regulatory protein of Neurospora crassa, binds upstream of nia, the tomato nitrate reductase gene, in vitro. Curr. Genet. 21:37-41.

Jaynes, R. A., and Elliston, J. E. 1980. Pathogenicity and canker control by mixtures of hypovirulent strains of Endothia parasitica in American chestnut. Phytopathology 70:453-456.

Kasahara, S., and Nuss, D. L. 1997. Targeted disruption of a fungal G-protein B subunit gene results in increased vegetative growth but reduced virulence. Mol. Plant-Microbe Interact. 10:984-993.

Kim, D. H., Rigling, D., Zhang, L., and Van Alfen, N. K. 1995. A new extracellular laccase of Cryphonectria parasitica is revealed by deletion of Lac1. Mol. Plant-Microbe Interact. 8:259-266.

Kim, M. J., Choi, J. W., Park, S. M., Cha, B. J., Yang, M. S., and Kim, D. H. 2002. Characterization of a fungal protein kinase from Cryphonectria parasitica and its transcriptional upregulation by hypovirus. Mol. Microbiol. 45:933-941.

Kim, M. J., Kwon, B. R., Park, S. M., Kim, J. M., Chung, H. J., Yang, M. S., Churchill, A. C. L., Van Alfen, N. K., and Kim, D. H. Promoter analysis of the cell surface-abundant and hypoviral-regulated cryparin gene from Cryphonectria parasitica. Mol. Cells. In press.

Kim, S., Leem, Y., Kim, K., and Choi, H. T. 2001. Cloning of an acidic laccase gene (clac2) from Coprinus congregatus and its expression by external pH. FEMS (Fed. Eur. Microbiol. Soc.) Microbiol. Lett. 195:151-156.

Kumar, S. V. S., Phale, P. S., Durani, S., and Wangikar, P. P. 2003. Combined sequence and structure analysis of the fungal laccase family. Biotechnol. Bioeng. 83:386-394.

Larson, T. G., Choi, G. H., and Nuss, D. L. 1992. Regulatory pathways governing modulation of fungal gene expression by a virulence-attenuating mycovirus. EMBO (Eur. Mol. Biol. Organ.) J. 11:4539-4548.

Law, C. J., and Timberlake, W. E. 1980. Developmental regulation of laccase levels in Aspergillus nidulans. J. Bacteriol. 144:509-517.

Leatham, G., and Stahmann, M. A. 1981. Studies on laccase of Lentinus edodes: Specificity, localization and association with the development of fruiting bodies. J. Gen. Microbiol. 125:147-157.

Lee, J. K., Tattar, T. A., Berman, P. M., and Mount, M. S. 1992. A rapid method for testing the virulence of Cryphonectria parasitica using excised bark and wood of American chestnut. Phytopathology 82:14541456 .

Marbach, I., Harel, E., and Mayer, A. M. 1985. Pectin, a second inducer for laccase production by Botrytis cinerea. Phytopathology 24:2559-2561.
Messerschmidt, A. 1997. Spatial structures of ascorbate oxidase, laccase and related proteins: Implications for the catalytic mechanism. Pages 23-80 in: Multi-Copper Oxidases. A. Messerschmidt, ed. World Scientific, Singapore.

Milgroom, M. G., and Cortesi, P. 2004. Biological control of chestnut blight with hypovirulence: A critical analysis. Annu. Rev. Phytopathol. 42:311-338.

Nielsen, H., Engelbrecht, S., Brunak, S., and Von, Heijine. G. 1997. Identification of prokaryotic and eukaryotic signal peptides and prediction of their cleavage sites. Protein Eng. 10:1-6.

Nuss, D. L. 1996. Using hypoviruses to probe and perturb signal transduction processes underlying fungal pathogenesis. Plant Cell 8:1845-1853.

Nuss, D. L. 2005. Hypovirulence: Mycoviruses at the fungal-plant interface. Nat. Rev. Microbiol. 3:632-642.

Palmieri, G., Giardina, P., Bianco, C., Fontanella, B., and Sannia, G. 2000. Copper induction of laccase isozymes in the lignolytic fungus Pleurotus ostreatus. Appl. Environ. Microbiol. 66:920-924.

Park, S. M., Choi, E. S., Kim, M. J., Cha, B. J., Yang, M. S., and Kim, D. H. 2004. Characterization of HOG1 homologue, CpMK1, from Cryphonectria parasitica and evidence for hypovirus-mediated perturbation of its phosphorylation in response to hypertonic stress. Mol. Microbiol. 51:1267-1277.

Perry, C. R., Matcham, S. E., Wood, D. A., and Thurston, C. F. 1993. The structure of laccase protein and its synthesis by the commercial mushroom Agaricus bisporus. J. Gen. Microbiol. 139:171-178.

Rigling, D., and Van Alfen, N. K. 1991. Regulation of laccase biosynthesis in the plant-pathogenic fungus Cryphonectria parasitica by doublestranded RNA. J. Bacteriol. 173:8000-8003.

Rigling, D., and Van Alfen, N. K. 1993. Extra- and intracellular laccases of the chestnut blight fungus, Cryphonectria parasitica. Appl. Environ. Microbiol. 59:3634-3639.

Rigling, D., Heiniger, U., and Hohl, H. R. 1989. Reduction of laccase activity in dsRNA-containing hypovirulent strains of Cryphonectria (Endothia) parasitica. Phytopathology 79:219-223.

Schouten, A., Wagemakers, L., Stefanato, F. L., Van der Kaaij, R. M., and Van Kan, J. A. 2002. Resveratrol acts as a natural profungicide and induces self-intoxification by a specific laccase. Mol. Microbiol. 43:883894.

Sengupta, N. D. S., and Mukherjee, M. 1997. Importance of laccase in vegetative growth of Pleurotus florida. Appl. Environ. Microbiol. 63:4120-4122.

Shang, J., Wu, X., Lan, X., Fan, Y., Dong, H., Deng, Y., Nuss, D. L., and Chen, B. 2008. Large-scale expressed sequence tag analysis for the chestnut blight fungus Cryphonectria parasitica. Fung. Genet. Biol. 45:319-327.

Smith, M., Shnyreva, A., Wood, D. A., and Thurston, C. F. 1998. Tandem organization and highly disparate expression of the two laccase genes lac 1 and lac 2 in the cultivated mushroom Agaricus bisporus. Microbiology 144:1063-1069.

Soden, D. M., and Dobson, A. D. W. 2001. Differential regulation of laccase gene expression in Pleurotus sajor-caju. Microbiology 147:17551763.

Temp, U., and Eggert, C. 1999. Novel interaction between laccase and cellobiose dehydrogenase during pigment synthesis in the white rot fungus Pycnoporus cinnabarinus. Appl. Environ. Microbiol. 65:389-395.

Thurston, F. 1994. The structure and function of fungal laccases. Microbiology 140:19-26.

Van Alfen, N. K., Jaynes, R. A., Anagnostakis, S. L., and Day, P. R. 1975. Chestnut blight: Biological control by transmissible hypovirulence in Endothia parasitica. Science 189:890-891.

Yaver, D. S., and Golightly, E. J. 1996. Cloning and characterization of three laccases genes from the white rot basidiomycete Trametes villosa: Genomic organization of the laccase gene family. Gene 181:95-102.

Yelton, M. M., Timberlake, W. E., and Van Den Hondel, C. A. M. J. J. 1985. A cosmid for selection genes by complementation in Aspergillus nidulans: Selection of the developmentally regulated yA locus. Proc. Natl. Acad. Sci. U.S.A. 82:834-838.

Yumiko, N., Sumiko, T., and Yasuhide, T. 2003. Method for analysis of tannic acid and its metabolites in biological samples : Application to tannic acid metabolism in the rat. J. Agr. Foodchem. 51:331-339. 